\title{
Examination of Wireless Power Transfer Combined with the Function of Distance Detection
}

\author{
Yinggang Bu, Masahiro Nishiyama, Takuto Ueda, Yoshimi Tashima, and Tsutomu Mizuno \\ Faculty of Engineering, Shinshu University, Nagano 380-8553, Japan
}

\begin{abstract}
Wireless power transfer using magnetic resonant coupling is expected to be widely used in the charging of an electric vehicle and in the use of home electric appliances. Wireless power transfer requires a high efficiency and power transfer over a long distance. However, the efficiency is reduced by the increase of the transmission distance and the change of the impedance of the receiving load. Therefore, a distance sensor using magnetic resonant coupling is proposed. In this paper, we propose a method of power transfer and distance detection using one couple of coils in real time. We also propose to use different frequencies in the power transfer and the distance detection, which are superposed and separated. Using the proposed approach, we demonstrated that the power transfer and the distance detection can be performed without relying on the change of the transmission distance.
\end{abstract}

Index Terms - Distance detection, separation, superposition, wireless power transfer.

\section{INTRODUCTION}

$\mathrm{W}$ IRELESS power transfer using magnetic resonant coupling has been more frequently researched recently because it provides the benefits of a long transmission distance and tolerance against position misalignment [1]-[4]. However, the transmission efficiency is reduced as the transmission distance changes and as position misalignment occurs [5]. Implementation of wireless power transfer requires the position information of the receiving coil to realize high efficiency transmission, to determine a range of power transfers, and to transfer power to moving objects.

Distance sensors using magnetic resonant coupling, which detect the distance between the transmitting and receiving coils, have been examined to determine the position information of the receiving coil [6][7]. Such distance sensors have the advantages of simple structure, low cost, resistance against environmental change, and long distance detection.

Therefore, we examined a circuit structure that combines the wireless power transfer capability using magnetic resonant coupling with distance sensing capability.

In this paper, we describe the circuit structure and examine the characteristics of the circuit of the power transfer and the distance detection in real time. This paper covers the following.

(1) Structure of a wireless power transfer with the function of distance detection

(2) Distance detection

(3) Power transfer

\section{Structure Of A Wireless Power TRANSFER Circuit COMBINED WITH THE FUNCTION OF DISTANCE DETECTION}

1) Structure of a wireless power transfer circuit combined with the function of distance detection

Fig. 1 shows the structure of wireless power transfer circuit combined with the function of distance detection. In this structure, both the power transfer and the detection of the

Manuscript received March 6, 2014. Corresponding author: M. Nishiyama (e-mail: 13tm237d@ shinshu-u.ac.jp).

Digital Object Identifier inserted by IEEE distance $l$ between the transmitting and receiving coils are performed simultaneously. To accomplish this simultaneous functionality, a frequency $f_{\mathrm{d}}$ for the distance detection and difference frequency $f_{\mathrm{p}}$ for power transfer are superposed on a pair of coils. The filter circuits 1-4 have the roles of superposition and separation of the different frequencies. The roles of each filter circuit are as follows.

(1) Distance detection

a) Filter circuits 1 and 2: The filter circuits 1 and 2 have the role of increasing the sensitivity of the distance detection. The filter circuit 1 and the transmitting coil resonate at $f_{\mathrm{d}}$ for a given distance between the transmitting coil and the receiving coil. In addition, the filter circuit 2 and the receiving coil resonate at $f_{\mathrm{d}}$ at the distance between transmitting coil and the receiving coil.

b) Filter circuits 3 and 4: The impedance of the filter circuits 3 and 4 are high at $f_{\mathrm{d}}$ so that the current $I\left(f_{\mathrm{d}}\right)$ for the distance detection is prevented from flowing into the circuit for power transfer.

\section{(2) Power transfer}

a) Filter circuits 1 and 2: The impedance of the filter circuits 1 and 2 are high at $f_{\mathrm{p}}$ so that the current $I\left(f_{\mathrm{p}}\right)$ for the power transfer is prevented from flowing into the circuit for distance detection.

b) Filter circuits 3 and 4: The filter circuits 3 and 4 have the role of increasing the sensitivity of the distance detection. The filter circuit 3 and the transmitting coil resonate at $f_{\mathrm{p}}$. In addition, the filter circuit 4 and the receiving coil resonate at $f_{\mathrm{p}}$.

The isolation transformer has the role of isolating the source for distance detection.

For the distance detection function, the input impedance $Z_{\mathrm{di}}$ of the source used for distance detection changes due to the changes in the magnetic flux crossing the receiving coil for different distances $l$. The distance detection voltage $V_{\mathrm{d}}$ changes due to the partial pressure of the internal impedance $Z_{0}(=50 \Omega)$ of the source used for distance detection and $Z_{\mathrm{di}}$. In this paper, $l$ is detected on the basis of $V_{\mathrm{d}}$, and the central axis of transmitting and receiving coils are the same axis.

It is possible to control $f_{\mathrm{p}}$ and the filter circuits using the information of distance so that the transmission efficiency is 


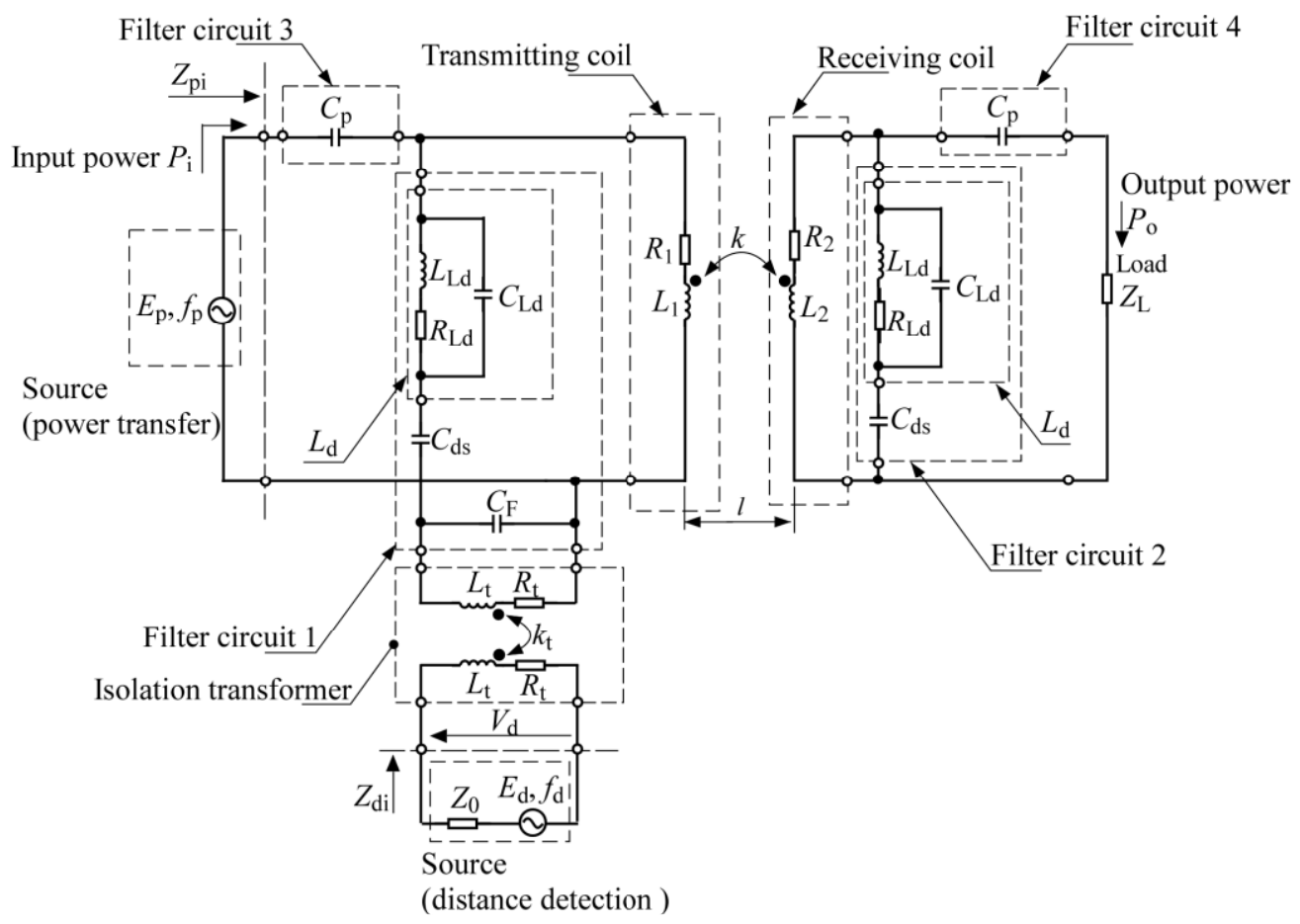

Fig. 1. Equivalent circuit of the wireless power transfer system with the function of distance detection.

optimized.

For the power transfer, the input power of the power transfer is $P_{\mathrm{i}}$, and the output power of the power transfer is $P_{\mathrm{o}}$. In addition, a supply voltage $E_{\mathrm{p}}$ used for the power transfer is adjusted as the amount of power transfer is varied from $P_{\mathrm{i}}=0$ $\mathrm{W}$ and $50 \mathrm{~W}$, and the load $Z_{\mathrm{L}}$ is fixed at $100 \Omega$.

2) Structure of the transmitting and receiving coils and their impedance characteristics

Common types of coils are spiral coil, solenoid coil, opened helical coil, and a coil that the iron core is inserted in each of types of coils [2]-[5]. As an example, the transmitting and receiving coils are spiral coils in this paper. The transmitting and receiving coils are each a spiral coil of the same structure. The outside diameter of the coils is $300 \mathrm{~mm}$, the number of turns is 40 and the winding pitch is $3 \mathrm{~mm}$. In addition, the number of strands of the Litz wire is 500, and each strand is a copper wire with a diameter of $0.07 \mathrm{~mm}$.

Fig. 2 shows the quality factor of the coils vs. the frequency characteristics. In addition, the resistance and the inductance values of the transmitting and receiving coils at $f=100 \mathrm{kHz}$ are $0.30 \Omega$ and $260 \mu \mathrm{H}$ and $0.33 \Omega$ and $275 \mu \mathrm{H}$, respectively. The impedance was measured using a Network Analyzer (Agilent, E5061B). In addition, the self-resonant frequency of the transmitting and receiving coils are $2.45 \mathrm{MHz}$. The individual difference of the impedance of the transmitting and receiving coils are less than $10 \%$. The impedance of the coils became unstable at frequencies of over $300 \mathrm{kHz}$ due to the self-resonant frequency; as a result, the power transfer is measured at a frequency of less than $300 \mathrm{kHz}$. In addition, the efficiency between the transmitting coil and the receiving coil depends on the quality factor and the coupling coefficient $k$. Therefore, the power transfer is performed in the frequency $f_{\mathrm{p}}$ range from $100 \mathrm{kHz}$ to $300 \mathrm{kHz}$ to increase the transmission

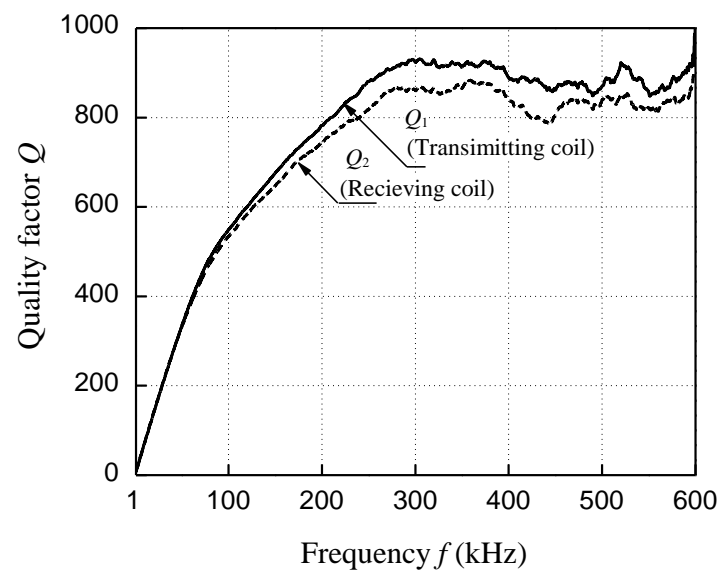

Fig. 2. Quality factor vs. frequency characteristics.

TABLE I

CiRCuit CONSTANTS

\begin{tabular}{llccc}
\hline \hline Item & & Symbol & Value & $($ Unit $)$ \\
\hline & Resistance & $R_{\mathrm{Ld}}$ & 7.8 & $(\Omega)$ \\
Filter & Inductor & $L_{\mathrm{Ld}}$ & 7.8 & $(\mathrm{mH})$ \\
circuit 1, 2 & Capacitor & $C_{\mathrm{ds}}$ & 34 & \\
& $C_{\mathrm{Ld}}$ & 0.106 & $(\mathrm{nF})$ \\
Filter & $C_{\mathrm{F}}$ & 1000 & \\
circuit 3, 4 & Capacitor & $C_{\mathrm{p}}$ & 3.3 & $(\mathrm{nF})$ \\
& Resistance & $R_{\mathrm{t}}$ & 4.6 & $(\Omega)$ \\
Isolation & Inductor & $L_{\mathrm{t}}$ & 3.4 & $(\mathrm{mH})$ \\
transformer & Coupling & $k_{\mathrm{t}}$ & 0.998 & - \\
& coefficient & $\mathrm{Z}_{\mathrm{L}}$ & 100 & $(\Omega)$ \\
Load & & & & \\
\hline \hline
\end{tabular}


efficiency. In addition, $f_{\mathrm{d}}$ is approximately $10 \mathrm{kHz}$, which is less than $1 / 10$ of $f_{\mathrm{p}}$ to enable the easy separation of the current for distance detection and the current for power transfer.

The resistances of the transmitting and receiving coils are $R_{1}$ and $R_{2}$, respectively, and inductances of the transmitting and receiving coils are $L_{1}$ and $L_{2}$, respectively, and the transmitting and receiving coils are magnetically coupled with a factor of $k$. The filter circuit 1 is comprised of capacitors $C_{\mathrm{ds}}$ and $C_{\mathrm{F}}$ and inductor $L_{\mathrm{d}}$. The equivalent circuit of the inductor is expressed by the resistance $R_{\mathrm{Ld}}$, the inductance $L_{\mathrm{Ld}}$ and the capacitance $C_{\mathrm{Ld}}$. The filter circuit 2 is comprised of capacitor $C_{\mathrm{ds}}$ and inductor $L_{\mathrm{d}}$. In addition, the filter circuits 3 and 4 are comprised of capacitance $C_{\mathrm{p}}$. The equivalent circuit of the isolation transformer is comprised of resistance $R_{\mathrm{t}}$, inductance $L_{\mathrm{t}}$ and coupling coefficient $k_{\mathrm{t}}$, with a load of $Z_{\mathrm{L}}$.

Table 1 presents the circuit constants at $f=10 \mathrm{kHz}$. $C_{\mathrm{ds}}$ and $L_{\mathrm{d}}$ are determined to be $34 \mathrm{nF}$ and $7.8 \mathrm{mH}$, respectively. In addition, $R_{\mathrm{Ld}}$ is $7.8 \Omega$, and the self-resonant frequency $f_{0}$ of $L_{\mathrm{d}}$ is $175 \mathrm{kHz} . f_{0}$ and $C_{\mathrm{Ld}}$ were calculated using the following equations.

$$
\begin{gathered}
f_{0}=\frac{1}{2 \pi} \sqrt{\frac{L_{\mathrm{Ld}}-C_{\mathrm{Ld}} R_{\mathrm{Ld}}{ }^{2}}{L_{\mathrm{Ld}}{ }^{2} C_{\mathrm{Ld}}}} \\
C_{\mathrm{Ld}}=\frac{L_{\mathrm{Ld}}}{\left(2 \pi f_{0} L_{\mathrm{Ld}}\right)^{2}+R_{\mathrm{Ld}}}
\end{gathered}
$$

In addition, the impedances of the filter circuits 1 and 2 are the highest at $175 \mathrm{kHz}$. Therefore, $C_{\mathrm{F}}$ for the filter circuit 1 is $1000 \mathrm{nF}$, and the low impedance at $f_{\mathrm{p}}$ is required to prevent the current for the power transfer from flowing into the circuit for distance detection. In addition, $C_{\mathrm{p}}$ is $3.3 \mathrm{nF}$. The isolation transformer is PT4 (OEP), and the load is $100 \Omega$.

\section{Distance Detection}

Impedance characteristics of the circuit were measured using a Network Analyzer (Agilent, E5061B). The power transfer and distance detection were simultaneously performed using the circuit shown Fig. 1. Because the variation of $Z_{\mathrm{di}}$ is the largest at the frequency of $9.8 \mathrm{kHz}$ in the distance range from $10 \mathrm{~mm}$ to $200 \mathrm{~mm}, f_{\mathrm{d}}$ was set to $9.8 \mathrm{kHz}$. Additionally, the supply voltage $E_{\mathrm{d}}$ for distance detection was fixed to $1 \mathrm{~V}$. Then, $f_{\mathrm{p}}$ was the frequency for which the power factor was the closest to 1 according to $l$.

Fig. 3 shows the distance detection voltage vs. distance characteristics for different values of the parameter of $P_{\mathrm{i}}$. According to Fig. $3, l$ was varied from $10 \mathrm{~mm}$ to $300 \mathrm{~mm}$, and $V_{\mathrm{d}}\left(P_{\mathrm{i}}=0 \mathrm{~W}\right)$ was varied from $310 \mathrm{mV}$ to $468 \mathrm{mV}$, at $P_{\mathrm{i}}=0 \mathrm{~W}$. The voltage applied to the circuit increases because $Z_{\mathrm{di}}$, which depends on $l$, increases. Additionally, the values of $V_{\mathrm{d}}\left(P_{\mathrm{i}}=0\right.$ $\mathrm{W})$ and $V_{\mathrm{d}}\left(P_{\mathrm{i}}=50 \mathrm{~W}\right)$ were $457.3 \mathrm{mV}$ and $455.7 \mathrm{mV}$ at $l=$ $100 \mathrm{~mm}$, respectively; thus, the difference of the voltage for the different input power values was $1.6 \mathrm{mV}$. The difference between $V_{\mathrm{d}}\left(P_{\mathrm{i}}=0 \mathrm{~W}\right)$ and $V_{\mathrm{d}}\left(P_{\mathrm{i}}=50 \mathrm{~W}\right)$ was the largest at $l=$ $200 \mathrm{~mm}$. The values of $V_{\mathrm{d}}\left(P_{\mathrm{i}}=0 \mathrm{~W}\right)$ and $V_{\mathrm{d}}\left(P_{\mathrm{i}}=50 \mathrm{~W}\right)$ were

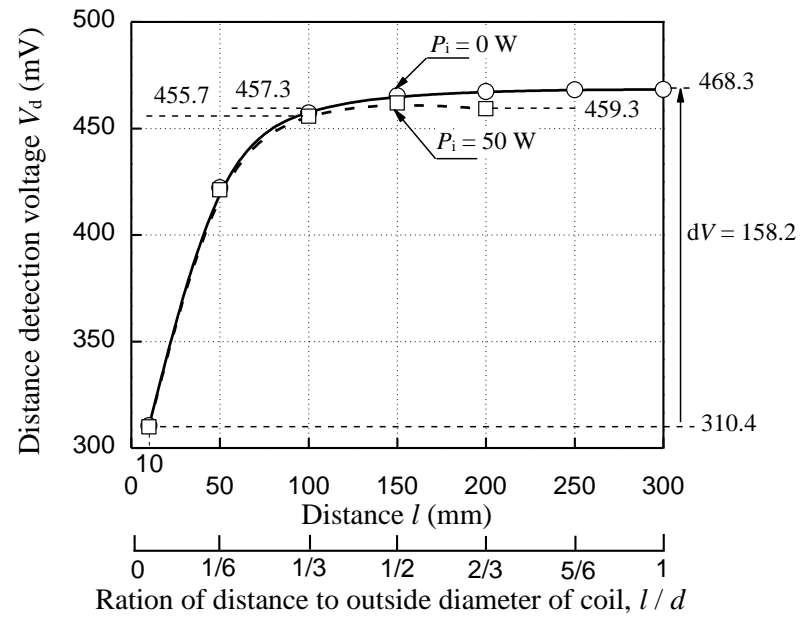

Fig. 3. Distance detection voltage vs. distance characteristics for different values of the parameter of $P_{\mathrm{i}}\left(E_{\mathrm{d}}=1 \mathrm{~V}, f_{\mathrm{d}}=9.8 \mathrm{kHz}\right)$.

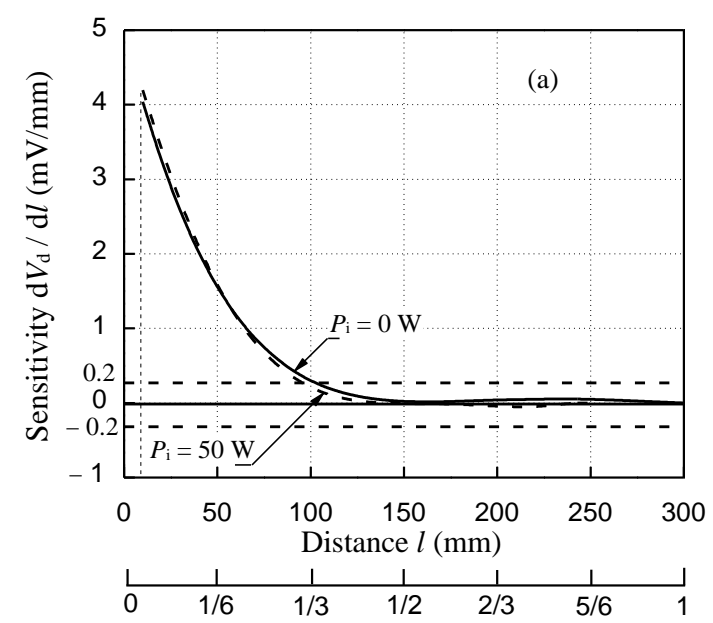

Ration of distance to outer diameter of coil, $l / d$

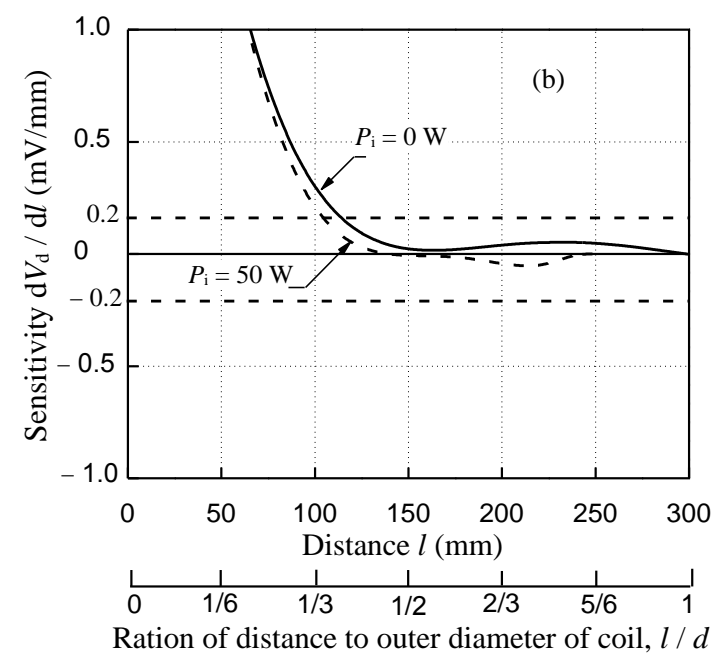

Fig. 4. (a) Sensitivity vs. distance characteristics for different values of the parameter of $P_{\mathrm{i}}\left(E_{\mathrm{d}}=1 \mathrm{~V}, f_{\mathrm{d}}=9.8 \mathrm{kHz}\right)$. (b) Zoom in the data shown in (a) from $-1.0 \mathrm{mV} / \mathrm{mm}$ to $1.0 \mathrm{mV} / \mathrm{mm}$. 
$467.3 \mathrm{mV}$ and $459.3 \mathrm{mV}$ at $l=200 \mathrm{~mm}$, respectively; thus, the difference was $8.0 \mathrm{mV}$. The factor that causes the difference between $V_{\mathrm{d}}\left(P_{\mathrm{i}}=0 \mathrm{~W}\right)$ and $V_{\mathrm{d}}\left(P_{\mathrm{i}}=50 \mathrm{~W}\right)$ to be the largest was impedance change due to the exothermic nature of the circuit elements. As a result, $V_{\mathrm{d}}$ changes as $Z_{\mathrm{di}}$ changes.

Fig. 4 shows the sensitivity vs. distance characteristics as a function of the parameter of $P_{\mathrm{i}}$. Figure 4 was calculated by differentiating a sixth-order polynomial that approximately describes the result in Fig. 3. Given a threshold voltage of 0.2 $\mathrm{mV}$ per $1 \mathrm{~mm}$, the circuit is able to determine the distance in the range from $10 \mathrm{~mm}$ to $100 \mathrm{~mm}$ at both $P_{\mathrm{i}}=0 \mathrm{~W}$ and $50 \mathrm{~W}$. Additionally, the distance detection error at $P_{\mathrm{i}}=0 \mathrm{~W}$ and $50 \mathrm{~W}$ was less than $8 \mathrm{~mm}$.

\section{POWER TRANSFER}

Fig. 5 shows the transmission efficiency vs. distance characteristics at $P_{\mathrm{i}}=1 \mathrm{~W}$. The efficiencies with the distance detection circuit and without the circuit were both greater than $80 \%$ in the distance range from $10 \mathrm{~mm}$ to $200 \mathrm{~mm}$. However, the loss increased with the significant decrease of the transmission efficiency in the distance range that $l$ is larger than $200 \mathrm{~mm}$. Therefore, the power transfer of $50 \mathrm{~W}$ was measured up to $l=200 \mathrm{~mm}$.

Fig. 6 shows the transmission efficiency vs. distance characteristics at $P_{\mathrm{i}}=50 \mathrm{~W}$. The efficiencies with the distance detection circuit and without the circuit were $86.8 \%$ and $84.1 \%$ at $l=200 \mathrm{~mm}$, respectively; the largest difference between the cases with and without distance detection was $2.7 \%$. Therefore, the decrease of the transmission efficiency by inserting the distance detection circuit can be ignored. In addition, as shown in Fig. 5 and Fig. 6, the decrease of the transmission efficiency for different input powers was $0.3 \%$ at most, and the cause of this decrease was the exothermic nature of the circuit elements.

\section{CONCLUSIONS}

In this paper, we proposed a wireless power transfer system combined with the function of distance detection, and the following aspects of the system were discussed.

\section{1) Distance detection}

A wireless power transfer system combined with the function of distance detection was proposed, which enabled the simultaneously performance of power transfer and distance detection. Given a threshold voltage of $0.2 \mathrm{mV}$ per $1 \mathrm{~mm}$, the system was found to be able to detect the distance in the range from $10 \mathrm{~mm}$ to $100 \mathrm{~mm}$ at $P_{\mathrm{i}}=0 \mathrm{~W}$ and $50 \mathrm{~W}$. In addition, the distance detection error at both $P_{\mathrm{i}}=0 \mathrm{~W}$ and $50 \mathrm{~W}$ was less than $8 \mathrm{~mm}$.

\section{2) Power transfer}

The power transfer was performed in the distance range from $10 \mathrm{~mm}$ to $200 \mathrm{~mm}$ at $P_{\mathrm{i}}=50 \mathrm{~W}$. The transmission efficiency was greater than $80 \%$ in the distance range from 10 $\mathrm{mm}$ to $200 \mathrm{~mm}$. The decrease of the transmission efficiency by inserting the distance detection circuit was up to $2.7 \%$. Therefore, we argued that the reduction of the efficiency by

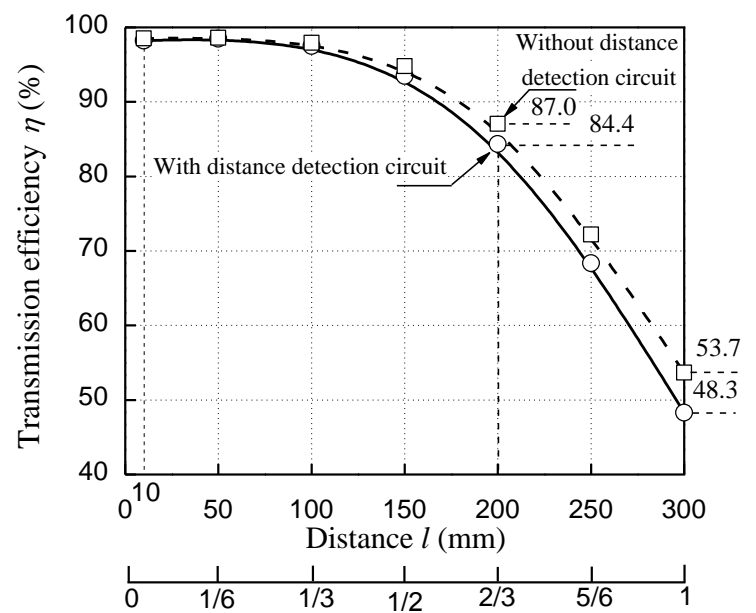

Ration of distance to outer diameter of coil, $l / d$

Fig. 5. Transmission efficiency vs. distance characteristics at $P_{\mathrm{i}}=1 \mathrm{~W}$.

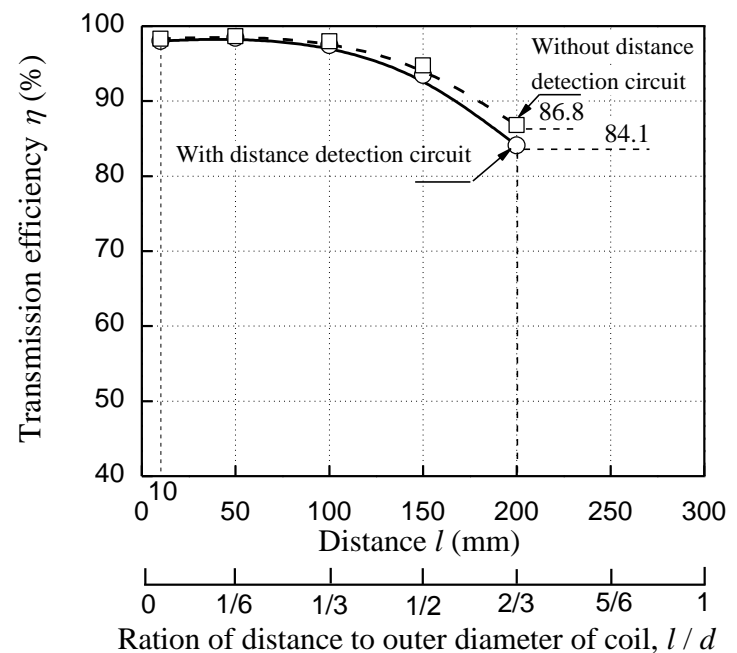

Fig. 6. Transmission efficiency vs. distance characteristics at $P_{\mathrm{i}}=50 \mathrm{~W}$.

inserting the distance detection circuit was minimal.

\section{REFERENCES}

[1] A Karalis, et. al., "Efficient wireless non-radiative mid-range energy transfer," Annals of Physics, vol. 323, pp. 34-48, 2008.

[2] K. Throngnumchi, T. Kai, Y. Minagawa, "A study on receiver circuit topology of a cordless battery charger for electric vehicles," IEEE Energy Conversion Congress and Exposition, pp. 843-850, Sep 2011.

[3] T. Imura, H. Okabe, Y. Hori, "Basic experimental study on helical antennas of wireless power transfer for electric vehicles by using magnetic resonant couplings," IEEE Vehicle Power and Propulsion Conference, pp. 936-940, Sep. 2009.

[4] T. Ishida, I. Awai, I. Sugiyama, "Measurement of resonator parameters for wireless power transmission system," Microwave Workshop Series on Innovative Wireless Power Transmission, pp. 211-214, May 2011.

[5] T. Mizuno, T. Ueda, S. Yachi, R.Ohtomo, Y. Goto, "Efficiency dependence on wire type for wireless power transfer of magnetic resonant coupling," Electrical Machines and Systems, pp. 1-4, Oct. 2012.

[6] G. Pirkl, K. Stockinger, and P. Lukowicz, "Adapting magnetic resonant coupling based relative positioning technology for wearable activitiy recogniton," Proc.IEEE Symposium on Wearable Computers, pp. 47-54, 2008.

[7] S. Hashi, S. Yabukami, H. Kanetaka, K. Ishiyama, K. I. Arai, "Wireless magnetic positioning-sensing system using optimized pickup coils for higher accuracy," IEEE Trans. Magn., vol. 47, pp. 3542-3545, 2011. 